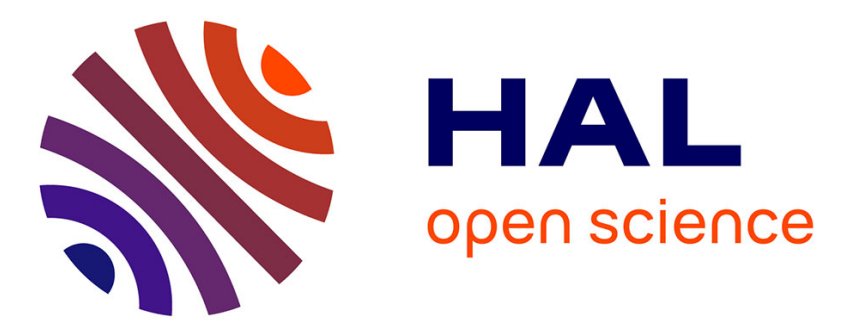

\title{
Persistent photoexcitation effect on the poly(3-hexylthiophene) film: Impedance measurement and modeling
}

Chang Hyun Kim, Krzysztof Kisiel, Jaroslaw Jung, Jacek Ulanski, Denis Tondelier, Bernard Geffroy, Yvan Bonnassieux, Gilles Horowitz

\section{To cite this version:}

Chang Hyun Kim, Krzysztof Kisiel, Jaroslaw Jung, Jacek Ulanski, Denis Tondelier, et al.. Persistent photoexcitation effect on the poly(3-hexylthiophene) film: Impedance measurement and modeling. Synthetic Metals, 2012, 162, pp.460. 10.1016/j.synthmet.2011.12.021 . hal-00670274

\section{HAL Id: hal-00670274 \\ https://hal-polytechnique.archives-ouvertes.fr/hal-00670274}

Submitted on 15 Feb 2012

HAL is a multi-disciplinary open access archive for the deposit and dissemination of scientific research documents, whether they are published or not. The documents may come from teaching and research institutions in France or abroad, or from public or private research centers.
L'archive ouverte pluridisciplinaire HAL, est destinée au dépôt et à la diffusion de documents scientifiques de niveau recherche, publiés ou non, émanant des établissements d'enseignement et de recherche français ou étrangers, des laboratoires publics ou privés. 


\title{
Persistent photoexcitation effect on the poly(3-hexylthiophene) film: Impedance measurement and modeling
}

\author{
Chang Hyun Kim ${ }^{\mathrm{a}, *}$, Krzysztof Kisiel ${ }^{\mathrm{b}}$, Jaroslaw Jung ${ }^{\mathrm{b}}$, Jacek Ulanski ${ }^{\mathrm{b}}$, Denis Tondelier ${ }^{\mathrm{a}}$, Bernard Geffroy ${ }^{\mathrm{a}, \mathrm{c}}$, Yvan Bonnassieux ${ }^{\mathrm{a}}$, \\ Gilles Horowitz ${ }^{\mathrm{a}}$
}

${ }^{a}$ LPICM, Ecole Polytechnique, CNRS, 91128 Palaiseau, France

${ }^{b}$ Department of Molecular Physics, Technical University of Lodz, Zeromskiego 116, 90-924 Lodz, Poland

${ }^{c}$ CEA Saclay, DSM/IRAMIS/SPCSI/LCSI, 91191 Gif-sur-Yvette, France

\begin{abstract}
We report on the equivalent circuit modeling of the relaxation behavior of an optically-excited thick poly(3-hexylthiophene) (P3HT) film by means of impedance spectroscopy. Fabricated metal-semiconductor-metal devices with Au electrodes showed a nearly perfect ohmic behavior under ambient conditions. Impedance measurements on illuminated P3HT device showed a dramatical decrease of the impedance modulus under illumination and very slow relaxation to the initial state. Impedance-frequency data obtained during relaxation could not be explained by a simple parallel resistance-capacitance circuit but it could be best fitted by incorporating a constant-phase element instead of a normal capacitance. By observing the variation of the circuit parameters, it is found that the relaxation process is dominated by slow recombination (elimination) of the excess photogenerated carriers, which is confirmed by the time-varying photoconductivity of the device.
\end{abstract}

Keywords: PH3T, Photosensitivity, Impedance spectroscopy, Equivalent circuit modeling

\section{Introduction}

Organic electronics is under constant progress and entering into viable applications such as light-emitting devices, flexible circuit arrays, or photovoltaic cells. A large number of organic semiconductors have been investigated in order to achieve optimum device performance. Among others, poly(3hexylthiophene) (P3HT), a hole-transporting polymer, can be undoubtedly considered as the most versatile organic material at the present time. By virtue of its promising physical property and reliable processibility, $\mathrm{P} 3 \mathrm{HT}$ is now adopted in almost all types of organic devices. P3HT can form well-ordered thin film phase with favored $\pi$-stacking resulting in high hole mobility and this is desirable for transistor architecture [1]. P3HT is also a highly photosensitive material with sufficient absorption of visible light and this enabled successful utilization of P3HT as an optical absorber in photovoltaic cells [2,3] or photodetectors $[4,5]$.

In spite of remarkable improvements of the P3HT-based devices, the material property of P3HT itself cannot be easily accessed or understood within complex devices because interface properties or (metal) contact features often dominate the operation of such devices. Furthermore, the correlation between electrical and optical properties are not straightforward due to various physical processes involved in optoelectronic devices like bulk heterojunction-based solar cells or photodiodes.

Here, we present a study on the optical/electrical properties of P3HT by analyzing simple metal-semiconductor-metal

*Corresponding author. Tel.: +3316933 4321; fax: +33169334333. E-mail address: chang-hyun.kim@polytechnique.edu (C. H. Kim).
(MSM) devices with a P3HT layer sandwiched between top and bottom Au electrodes. By measuring the frequency-dependent impedance with light exposure, we could observe a significant change of the ac-response of MSM device under illumination and a slow relaxation to the initial state without any permanent effect. Good ohmic contacts and utilization of thick P3HT films $(\sim 10 \mu \mathrm{m})$ were advantageous to minimize injection-dominated effect and/or interface-related phenomena, so we could focus on the bulk property of the P3HT device. The device under relaxation could be well modeled through an equivalent circuit composed of a resistance and a constant-phase element in parallel. The persistent photoexciation effect could be interpreted by an exponential decrease of the photoconductivity of the bulk film with considerable lifetime (110 minutes), which could be accounted for by a slow detrapping process in the presence of a high density of traps without any high electric field applied.

\section{Experimental}

MSM devices were fabricated on a Cr-coated steel substrate cleaned with ultrasonication in chloroform. One hundred fifty nanometers of Au bottom electrode was first vacuumevaporated on the substrate. PH3T (provided by American Dye Source, Inc.) was dissolved in chloroform at a concentration of $8 \mathrm{mg} / \mathrm{mL}$ and drop-casted on the bottom electrode. The film was then dried at $50{ }^{\circ} \mathrm{C}$ for 24 hours in a vacuum oven. This process gave relatively thick P3HT films with a measured thickness of $\sim 10 \mu \mathrm{m}$. The Au top electrode $(150 \mathrm{~nm})$ was finally deposited by vacuum-evaporation with a defined active area of $0.78 \mathrm{~cm}^{2}$. 


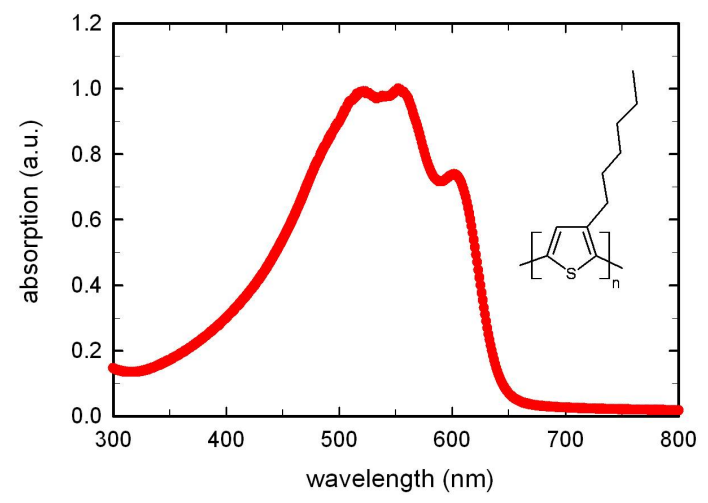

Figure 1: Abosrption spectrum of the PH3T film. Inset: molecular structure of P3HT

UV-VIS absorption spectra of P3HT films were recorded using a double beam UV-VIS-NIR Varian Inc. spectrometer model Carry 5000. Current-voltage $(I-V)$ measurements were carried out using a Keithley 2400 source meter in the dark. Impedance data were obtained under illumination or in the dark using broadband dielectric spectroscopy system (Novocontrol) including high resolution dielectric analyzer ALPHA-ANB in a broad frequency range $\left(10^{-2}\right.$ to $\left.10^{7} \mathrm{~Hz}\right)$. The sample was illuminated by a white light fluorescent lamp under ambient atmosphere.

\section{Results and discussion}

\subsection{Film and device properties}

Figure 1 shows an absorption spectrum of the P3HT film with the chemical structure of P3HT. As well known from previous studies, the P3HT layer absorbs large portion of the visible photons (roughly from $400 \mathrm{~nm}$ to $700 \mathrm{~nm}$ ) [3, 6]. Making use of this broad absorption spectrum, Antognazza et al. could recently demonstrate the selective color sensor based on P3HT diodes exploiting the internal filter effect [5]. By estimating the wavelength at the absorption edge in Figure $1(645 \mathrm{~nm})$, it is possible to calculate the optical bandgap of P3HT. The resulting optical bandgap is $1.9 \mathrm{eV}$, in good accordance with the literature [7].

An MSM-type organic device can behave either as a (diodelike) rectifier or a resistor, mostly determined by the injection energy barrier at metal/organic interfaces $[8,9]$. If one refers to the literature value of the work function of $\mathrm{Au}$ as $\sim 5.0 \mathrm{eV}$ $[10,11]$ and the HOMO level of P3HT as $~ 5.1 \mathrm{eV}$ [6], our symmetric $\mathrm{Au} / \mathrm{P} 3 \mathrm{HT} / \mathrm{Au}$ device is expected to have ohmic contacts (for hole-only conduction) with negligible contact resistance. Figure 2 provides notable aspects of the electrical behavior of the P3HT device. The $I-V$ curves shown were measured under low vacuum $\left(2 \times 10^{-2} \mathrm{mbar}\right)$ and ambient atmosphere. The two curves assure satisfying symmetry and linearity throughout the measurement range, thus confirming resistor-like behavior with ohmic contacts at both electrodes. Moreover, it can be stated from Figure 2 that an exposure to the ambient air resulted in an increase of the conductivity (higher slope) by a factor of 2.2 and an enhanced ohmic feature (better linearity).

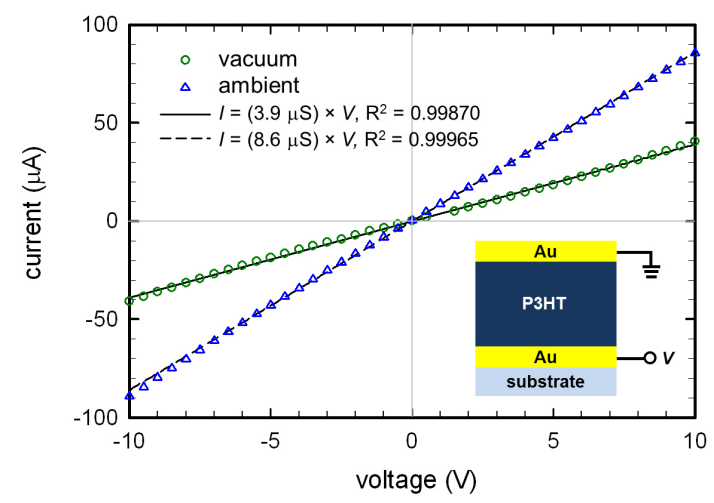

Figure 2: Current-voltage characteristics of the Au/P3HT/Au device with ambient effect on the electrical behavior. Inset: vertical type MSM device structure. Applied voltage is on the bottom Au electrode with the top electrode grounded. linear-regression lines are drawn with the equations that quantitatively show the increase of the slope and the linearity at ambient air.

The improved conductivity due to air exposure could be explained in terms of oxygen doping, which is a widely accepted idea at the present time [12, 13, 14]; oxygen molecules can be adsorbed and diffuse into the organic solid and they can serve as efficient p-type dopants (acceptors) by redox reaction via their molecular orbitals. The enhanced ohmic contact could be regarded then as a concomitant event with oxygen doping. Even though it seems marginal, an energy mismatch between the Fermi levels of Au and P3HT could lead to the formation of a depletion region and this is a possible cause of the slightly non-ohmic $I-V$ curve at low-voltage under vacuum. Higher carrier density is expected when ambient doping takes place and these additional carriers could reduce the depletion width down to a negligible scale and/or compensate other interface-related parasitic (non-ohmic) effects [15].

\subsection{Impedance spectroscopy}

Impedance spectroscopy is a well-developed technique, especially in the field of electrochemical analysis of electrolytes and interfaces $[16,17]$. The practical usefulness on the analysis of solid-state electronic devices is that it allows to describe an equivalent circuit of the given system and thus to understand physical/electrical processes under various conditions $[8,18,19]$.

Figure 3 shows the results of impedance spectroscopy on a P3HT MSM device plotted in Nyquist diagram (real part versus -imaginary part). Upon light exposure, the device responded quickly and came to the state in which the overall impedance modulus is extremely reduced compared to the initial state. At this point, it is worth reminding that the impedance is always given as a complex number and the modulus corresponds to the distance from the origin to each measurement point on a complex plane Figure 3. Surprisingly, this excited state did not immediately recover towards its initial state after turning off the light but the relaxation took several hours as monitored by repeated measurements shown in Figure 3. It turned out that in spite of this extraordinarily slow relaxation (or persistent photoexcitation effect), the device could fully relax to its 


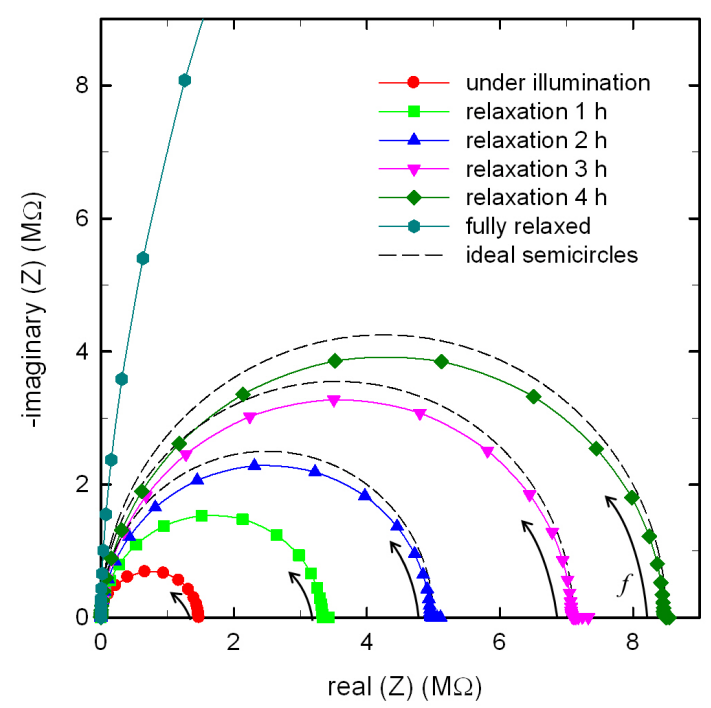

Figure 3: Nyquist diagram containing the frequency-dependent impedance data under light exposure and under relaxation to the initial state. The applied dc voltage is $0 \mathrm{~V}$ and the amplitude of small-signal (ac) voltage is $0.01 \mathrm{~V}$. The arrows emanating from the real axis indicate the direction of increasing smallsignal frequency (from $10^{-2}$ to $10^{7} \mathrm{~Hz}$ ). Three ideal semicircles are drawn to help recognize the non-ideality of the data curves; the height of the experimental arc is smaller than the half-width.

initial state so that the excitation/relaxation process could be described as reversible.

One can make sure of the photostability of our P3HT device by taking the experimental and geometrical factors into consideration. First, the illumination time on the device was 1 hour and this is not long enough to provoke any serious photoinduced chemical change. Photodegradation studies on P3HT revealed that continuous illumination (typically more than several tens of hours) can result in the formation of degradation species such as sulfoxides and sulfinate esters and a loss of $\pi$ conjugation $[20,21]$. Second, the expected photon penetration depth is of the order of hundred nanometers (one percent of the film thickness in our case) so that most of the bulk film is not subject to the direct photo-absorption [22, 23]. In other words, even though the charge transport and electrical conduction occur throughout the whole film, the effective photogeneration is limited to a thin top surface region.

Note that we focus on the 'relaxation' process itself in the whole study and thus Figure 3 is not scaled with an entire feature of the fully relaxed (or initial) state. As mentioned above, owing to the high photosensitivity of P3HT, the impedance (modulus) of the unexposed state is much higher than that of the illuminated state. On that account, the trajectory of the 'fully relaxed' data in Figure 3 extends very far out of the region shown, intercepting the real axis at $91 \mathrm{M} \Omega$.

\subsection{Equivalent circuit model}

Impedance modeling generally starts with a choice of the equivalent circuit of the system that can be usually estimated from the shape of the data curve in Nyquist (or complex) plot. The measured data is then fitted to the selected circuit in order to extract each circuit parameter of the circuit. The sim- (a)

(b)
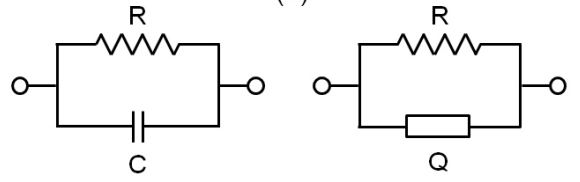

(c)

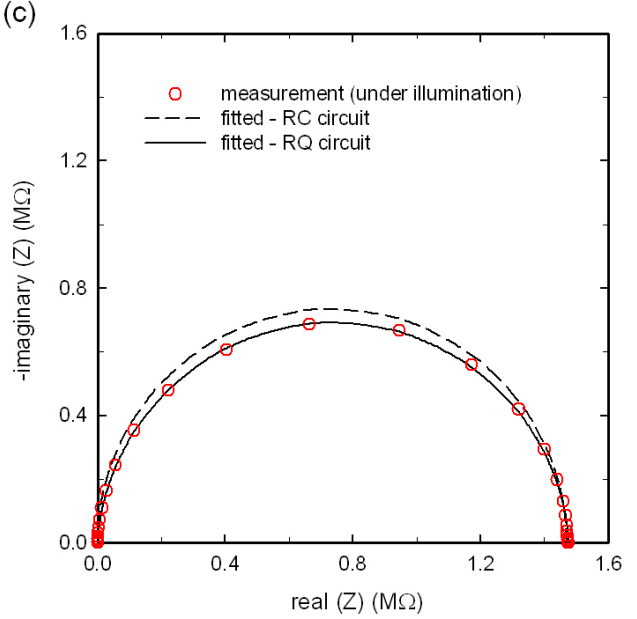

Figure 4: (a) Parallel resistance and capacitance circuit (RC circuit). (b) Parallel resistance and CPE (constant-phase element) circuit (RQ circuit). (c) Fitted experimental data to both RC and RQ equivalent circuits.

plest case is the single parallel resistance/capacitance (RC) circuit and the corresponding impedance data features a single perfect semicircle on the complex plane [8, 24]. If the given impedance data contains two or more intermixed semicircles, there is a need to draw a second or third RC element in the circuit [19, 17].

The data curves in Figure 3 look like ideal semicircles but with careful examination, it can be recognized that there exist slight distortions. Figure 4 shows the comparison of two suggested equivalent circuits; this emphasizes the central importance of a proper selection of the circuit prior to fitting experimental data. It is worth presenting some mathematical formulations to help thoroughly understand the proposed circuits.

The impedance $(Z)$ is defined as the ratio of the phasor voltage and the phasor current measured in ohms $(\Omega)$ and the impedance of a resistance $\left(Z_{R}\right)$ and a capacitance $\left(Z_{C}\right)$ are expressed as [25]:

$$
Z_{R}=R, \quad Z_{C}=\frac{1}{j \omega C},
$$

where $R$ is the resistance, $j$ the imaginary unit, $\omega$ the angular frequency of the applied ac small-signal, and $C$ the capacitance.

The total impedance of a RC circuit [Figure 4(a)] $\left(Z_{R C}\right)$ can be then simply obtained by the combination rule:

$$
Z_{R C}=\frac{1}{\frac{1}{Z_{R}}+\frac{1}{Z_{C}}}=\frac{R}{1+j \omega R C}
$$

If we manipulate Equation (2) to get separate real and imaginary part of $Z_{R C}$, it comes to:

$$
\operatorname{Re}\left(Z_{R C}\right)=\frac{R}{1+\omega^{2} R^{2} C^{2}},
$$




$$
\operatorname{Im}\left(Z_{R C}\right)=-\frac{\omega R^{2} C}{1+\omega^{2} R^{2} C^{2}} .
$$

Here, $\operatorname{Re}\left(Z_{R C}\right)$ and $\operatorname{Im}\left(Z_{R C}\right)$ represent the real and imaginary part of $Z_{R C}$, respectively. According to Equations (3) and (4), the response of a $\mathrm{RC}$ circuit marks a semicircle on the complex plane. There is a characteristic frequency $\left(\omega_{R C}\right.$ or $\left.f_{R C}\right)$ that produces the minimum value of $\operatorname{Im}\left(Z_{R C}\right)$ and can be obtained by the first derivative with respect to $\omega$ :

$$
\left.\frac{d \operatorname{Im}\left(Z_{R C}\right)}{d \omega}\right|_{\omega=\omega_{R C}}=0 \quad \text { with } \quad \omega_{R C}=2 \pi f_{R C}=\frac{1}{R C} .
$$

Note from Equation (5) that $\omega_{R C}$ contains the information of the two circuit parameters $R$ and $C$. In practice, $\omega_{R C}$ (or $f_{R C}$ ) can be read from the top point of the semicircle [as normally the $y$ axis in a Nyquist digram is the 'negative' $\operatorname{Im}(Z)]$.

When the experimental curve was fitted to this RC circuit, a fine fit was obtained but it is not a consummate model for the system [Figure 4(c)]. We found out that such a depressed circular arc can be more appropriately modeled by replacing the capacitor by a constant-phase element (CPE) [26].

The impedance of a $\mathrm{CPE}\left(Z_{Q}\right)$ is defined as:

$$
Z_{Q}=\frac{1}{(j \omega)^{\alpha} Q}
$$

Here $\alpha$ is the characteristic exponent that indicates the degree of imperfection of a (realistic) capacitive element $(0<\alpha \leq 1)$. $\alpha$ equally determines the phase shift of a CPE that is independent of $\omega$ (thus the 'constant' phase) and lies between 0 and $-\frac{\pi}{2}$ rad. $Q$ has a unit of $\mathrm{F} \cdot \mathrm{s}^{\alpha-1}$ and thus depends on $\alpha$. It has a close meaning to the capacitance $C$ in $Z_{C}$ (further discussion will be given in the next Section).

The total impedance of an RQ circuit [Figure 4(b)] $\left(Z_{R Q}\right)$ and its real part $\left[\operatorname{Re}\left(Z_{R Q}\right)\right]$ and imaginary part $\left[\operatorname{Im}\left(Z_{R Q}\right]\right.$ are deduced as follows:

$$
\begin{gathered}
Z_{R Q}=\frac{1}{\frac{1}{Z_{R}}+\frac{1}{Z_{Q}}}=\frac{R}{1+(j \omega)^{\alpha} R Q}, \\
\operatorname{Re}\left(Z_{R Q}\right)=\frac{R\left[1+\omega^{\alpha} R Q \cos \left(\frac{\alpha \pi}{2}\right)\right]}{1+2 \omega^{\alpha} R Q \cos \left(\frac{\alpha \pi}{2}\right)+\omega^{2 \alpha} R^{2} Q^{2}}, \\
\operatorname{Im}\left(Z_{R Q}\right)=-\frac{\omega^{\alpha} R^{2} Q \sin \left(\frac{\alpha \pi}{2}\right)}{1+2 \omega^{\alpha} R Q \cos \left(\frac{\alpha \pi}{2}\right)+\omega^{2 \alpha} R^{2} Q^{2}} .
\end{gathered}
$$

Note that when $\alpha=1$, a CPE reduces back to a normal capacitance and $Z_{Q}$ becomes identical to $Z_{C}$ (with only $C$ replaced by $Q)$. However, with $\alpha \neq 1$, one can predict, by Equations (8) and (9), that the complex-plane representation of $Z_{R Q}$ will result in a depressed semicircle. As shown in Figure 4(c), the RQ circuit is a more reliable choice for fitting our experimental impedance data. The extracted $\alpha$ is 0.96 here.

The characteristic frequency of a RQ circuit $\left(\omega_{R Q}\right.$ or $\left.f_{R Q}\right)$ can now be defined in a similar way to the RC circuit:

$$
\left.\frac{d \operatorname{Im}\left(Z_{R Q}\right)}{d \omega}\right|_{\omega=\omega_{R Q}}=0 \text { with } \omega_{R Q}=2 \pi f_{R Q}=\frac{1}{(R Q)^{\frac{1}{\alpha}}} .
$$

Table 1: Summary of the circuit parameters during relaxation

\begin{tabular}{ccccc}
\hline State & $\alpha$ & $R[\mathrm{M} \Omega]$ & $Q\left[\mathrm{pF} \cdot \mathrm{s}^{\alpha-1}\right]$ & $C_{\text {eff }}[\mathrm{pF}]$ \\
\hline Illuminated & 0.96 & 1.5 & 340 & 250 \\
1h relaxed & 0.94 & 3.4 & 380 & 260 \\
2h relaxed & 0.95 & 5.0 & 370 & 260 \\
3h relaxed & 0.95 & 7.1 & 360 & 260 \\
4h relaxed & 0.95 & 8.5 & 350 & 260 \\
Fully relaxed & 0.96 & 91 & 290 & 250 \\
\hline
\end{tabular}

Note that, as in the RC circuit, $\omega_{R Q}$ is uniquely determined by the three circuit parameters $R, Q$, and $\alpha$.

The incorporation of CPE in the circuit system has been generally intended to characterize non-ideal capacitance. Previously proposed physical arguments on CPE comprise distribution of energy states, spatially distributed conductivity, dispersive (frequency-dependent) dielectric property, etc [27, 28]. Hence, the observation of a CPE in our result from the P3HT device can most probably be attributed to the inhomogeneous contact interface(s) between the highly rough polymeric P3HT surface and the metal electrode.

\subsection{Circuit parameters during relaxation}

By adopting the RQ circuit, the device under illumination and under relaxation (all data curves in Figure 3 ) could be accurately fitted with precise circuit parameters $(R, Q$, and $\alpha)$. Now it is possible to analyze the variation of each parameter as a function of time after light exposure. The parameter $Q$, however, is a somewhat ambiguous quantity in that its unit $\left(\mathrm{F} \cdot \mathrm{s}^{\alpha-1}\right)$ varies with $\alpha$. In other words, it is difficult to argue over changing $Q$ values if its unit changes as well.

One strategy can be applied to transcend this limitation, focusing on the response of the P3HT system at the characteristic frequency $\omega_{R Q}$. We can define the effective capacitance $C_{\text {eff }}$ (of the whole RQ system) that has a unit of an ordinary capacitance. The definition is done by finding an (effective) RC circuit that features the same characteristic frequency as that of the RQ circuit $\left(\omega_{R Q}\right)$. Referring to Equations (5) and (10), this statement is expressed as:

$$
\omega_{R Q}=\frac{1}{(R Q)^{\frac{1}{\alpha}}}=\frac{1}{R C_{\mathrm{eff}}} .
$$

The above Equation readily gives $C_{\text {eff }}$ as:

$$
C_{\mathrm{eff}}=R^{\frac{1}{\alpha}-1} Q^{\frac{1}{\alpha}}
$$

Note that $C_{\text {eff }}$ is uniquely determined in any given RQ circuit. Effectively, $C_{\text {eff }}$ is a parameter that keeps any non-ideal capacitive characteristics of the RQ system and enables practical analysis in the capacitance unit.

Table 1 lists fitted $\alpha, R, Q$ with calculated $C_{\text {eff }}$ and Figure 5 shows the variation of $R$ and $C_{\text {eff }}$ during relation process. There is no significant variation of $\alpha$ under excitation and relaxation. From this fact, it can be inferred that the degree of deformation of the CPE is not seriously affected by the light exposure. This seems logical as the physical meaning of the CPE was mainly 

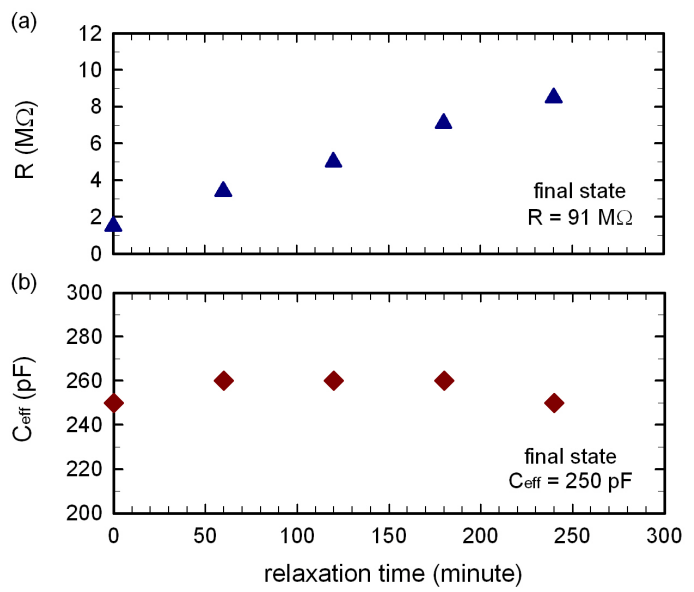

Figure 5: Variation of the circuit parameters of the P3HT MSM device: (a) resistance $R$ and (b) effective capacitance $C_{\text {eff }}$. Fitting errors were negligibly small and not visible when indicated with error bars. Final (or relaxed) state value is also indicated in each panel.

attributed to the geometrical problem. In contrast, it is found that the resistance $R$ is dramatically reduced under light and gradually increases towards its final state value. The decrease of $R$ could be interpreted as the increase of the conductivity by photogenerated (excess) charge carriers. $C_{\text {eff }}$ is practically constant under illumination/relaxation process so that we can think of this capacitance as the geometrical capacitance $C_{\mathrm{g}}$ that is defined by geometrical overlap of two metal electrodes and is not modified by external light exposure. Additionally, it is possible to estimate the dielectric constant of the P3HT film by using the parallel-plate capacitor equation:

$$
C_{\text {eff }, \mathrm{av}}=C_{\mathrm{g}}=k \epsilon_{0} \frac{A}{L},
$$

where $C_{\text {eff,av }}$ is the average $C_{\text {eff }}(257 \mathrm{pF}), k$ the dielectric constant of P3HT, $\epsilon_{0}$ the permittivity of vacuum, $A$ the active area of the device $\left(0.78 \mathrm{~cm}^{2}\right)$, and $L$ the film thickness $(9.6 \mu \mathrm{m})$. Calculated dielectric constant $k$ of P3HT is 3.6 , which would correspond to the quasistatic value [8].

From the above analysis on the circuit parameters, it is possible to tactically simplify the impedance response to a variation of the (bulk) resistance in P3HT as there was no critical influence of light on either $\alpha$ or $C_{\text {eff }}$. At this point, we can regard our MSM device as a photoconductor owing to its ohmic conduction (Figure 2) with photosensitive resistance (Figure 5). In order to quantify the photoconductivity $\left(\sigma_{\mathrm{ph}}\right)$, we can first convert the extracted resistances $(R)$ to the conductivities $(\sigma)$ by taking the geometrical factors into account:

$$
\sigma=\frac{1}{R} \times \frac{L}{A}
$$

$\sigma_{\mathrm{ph}}$ is then simply calculated by:

$$
\sigma_{\mathrm{ph}}=\sigma_{\text {illu }}-\sigma_{\text {dark }},
$$

where $\sigma_{\text {illu }}$ indicates each conductivity value under illumination/relaxation and $\sigma_{\text {dark }}$ is the dark conductivity calculated

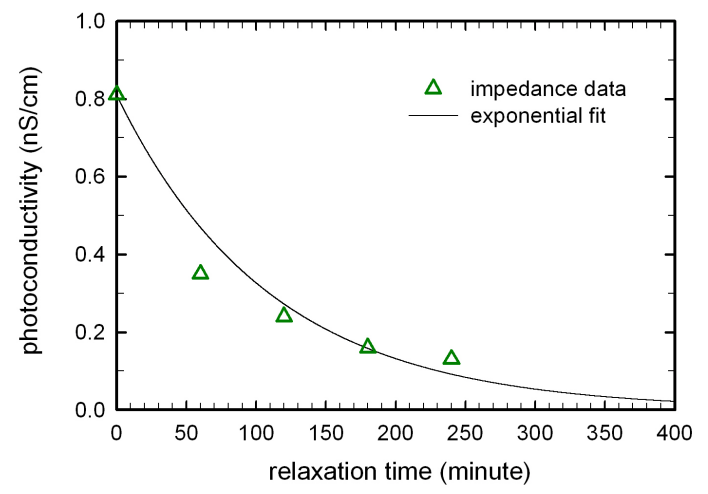

Figure 6: Photoconductivity plotted as a function of time. The calculated values are compared with the exponential fit that can account for the recombination of the excess carriers with a fixed decay time constant.

from the resistance at the final (or fully relaxed) state. Here, it is worth clarifying that even though $\sigma_{\text {dark }}$ is deduced from the fully-relaxed state, it can also represent the unillumianated (or dark) state since the excitation/relaxation process was reversible under given conditions.

Figure 6 shows the variation of $\sigma_{\mathrm{ph}}$ as a function of relaxation time. From conventional generation/recombination kinetics, it is expected that the photogenerated electron-hole pairs recombine with a carrier-density-dependent recombination rate at every instance and this results in an exponential decay of the photoconductivity expressed as [29]:

$$
\sigma_{\mathrm{ph}}(t)=\sigma_{\mathrm{ph}, 0} \exp \left(-\frac{t}{\tau_{d}}\right)
$$

Here, $\sigma_{\mathrm{ph}}(t)$ is the time-dependent photoconductivity of the P3HT layer, $\sigma_{\mathrm{ph}, 0}$ the photoconductivity under illumination (when the relaxation begins), and $\tau_{d}$ the decay time constant. The best fit curve shown in Figure 6 gives $\tau_{d}=110$ minutes.

To our knowledge, such an extremely slow relaxation behavior (or light-induced memory effect) of the P3HT film in MSM configuration has not been easily observed elsewhere. Previously, photocapacitance measurements of the P3HT-based MIS (metal-insulator-semiconductor) device showed prolonged recovery of the modulated capacitance due to trapped minority carriers at the interface states [30, 31]. More recently, Kim et al. explained the persistent photocurrent in dihexylquarterthiophene (DH4T) organic transistor with a slow detrapping of photogenerated carriers and they observed that the gate electric field could accelerate this detrapping process [32]. A similar phenomenon was reported by Lutsyk et al. on the study of poly(3-octylthiophene) (P3OT) transistor and they also emphasized the role of gate bias for erasing the memory effect [33]. Despite the dissimilarities in the material and/or the device geometry, it is likely that these results can help interpret our observation. Note that our impedance spectroscopy results in Figure 3 are obtained under dc bias of $0 \mathrm{~V}$ and a P3HT film with disordered polymer network should contain high density of defect or trap sites. With these two factors combined, photogenerated carriers could be easily trapped in the bulk film and their detrapping would take long time without assisting high 
(dc) electric field applied. Then, the slow detrapping of carriers results in a slow recombination and in turn, leads to the persistent effect on the device.

\section{Conclusion}

In this study, we presented a long-lasting photoexcitation effect on the P3HT-based metal-semiconductor-metal (MSM) device. It was revealed that the impedance characteristics of this ohmic-type device were significantly modulated upon light exposure. Impedance data were well fitted by an equivalent circuit containing a constant-phase element (CPE) that accommodates the non-ideal capacitor due to geometrical inhomogeneity. Impedance modeling allowed us to simplify the slow relaxation behavior to an exponential decay of the photoconductivity with an extended time constant (110 minutes). This result was then attributed to the slow detrapping of photogenerated carriers in the presence of trapping sites without high electric field. We believe that the phenomenon reported here can characterize intrinsic (or natural) physical property of the material itself without any possible accelerating factor imposed on the natural relaxation process.

\section{Acknowledgments}

This work was supported by NoE PolyNet from the European Community's Seventh Framework Program (FP7/20072013) under Grant No. 214006. C. H. Kim wishes to thank the Vice Presidency for External Relations (DRE) in Ecole Polytechnique for the Ph.D. fellowship.

\section{References}

[1] R. A. Street, Adv. Mater. 21 (2009) 2007.

[2] P. Vanlaeke, A. Swinnen, I. Haeldermans, G. Vanhoyland, T. Aernouts, D. Cheyns, C. Deibel, J. D’Haen, P. Heremans, J. Poortmans, J. Manca, Sol. Energy Mater. Sol. Cells 90 (2006) 2150.

[3] V. D. Mihailetchi, H. X. Xie, B. de Boer, L. J. A. Koster, P. W. M. Blom, Adv. Funct. Mater. 16 (2006) 699.

[4] T. Rauch, M. Boberl, S. F. Tedde, J. Furst, M. V. Kovalenko, G. Hesser, U. Lemmer, W. Heiss, O. Hayden, Nat. Photonics 3 (2009) 332.

[5] M. R. Antognazza, D. Musitelli, S. Perissinotto, G. Lanzani, Org. Electron. 11 (2010) 357.

[6] J. Y. Kim, K. Lee, N. E. Coates, D. Moses, T.-Q. Nguyen, M. Dante, A. J. Heeger, Science 317 (2007) 222.

[7] G. Dennler, M. C. Scharber, C. J. Brabec, Adv. Mater. 21 (2009) 1323.

[8] C. H. Kim, O. Yaghmazadeh, D. Tondelier, Y. B. Jeong, Y. Bonnassieux, G. Horowitz, J. Appl. Phys. 109 (2011) 083710.

[9] D. Braga, N. Battaglini, A. Yassar, G. Horowitz, M. Campione, A. Sassella, A. Borghesi, Phys. Rev. B 77 (2008) 115205.

[10] F. Amy, C. Chan, A. Kahn, Org. Electron. 6 (2005) 85.

[11] N. Koch, A. Kahn, J. Ghijsen, J. J. Pireaux, J. Schwartz, R. L. Johnson, A. Elschner, Appl. Phys. Lett. 82 (2003) 70.

[12] O. D. Jurchescu, J. Baas, T. T. M. Palstra, Appl. Phys. Lett. 87 (2005) 052102.

[13] S. Ogawa, T. Naijo, Y. Kimura, H. Ishii, M. Niwano, Appl. Phys. Lett. 86 (2005) 252104.

[14] M. L. Chabinyc, R. A. Street, J. Northrup, Appl. Phys. Lett. 90 (2007) 123508.

[15] R. S. Muller, T. I. Kamins, M. Chan, Device Electronics for Integrated Circuits, Wiley, New York, 3rd edition, 2003.

[16] J. R. Macdonald, Ann. Biomed. Eng. 20 (1992) 289.
[17] Q. Wang, J.-E. Moser, M. Gratzel, J. Phys. Chem. B 109 (2005) 14945.

[18] J. Drechsel, M. Pfeiffer, X. Zhou, A. Nollau, K. Leo, Synth. Met. 127 (2002) 201.

[19] D. Braga, M. Campione, A. Borghesi, G. Horowitz, Adv. Mater. 22 (2010) 424.

[20] M. Manceau, A. Rivaton, J.-L. Gardette, S. Guillerez, N. Lemaitre, Polym. Degrad. Stabil. 94 (2009) 898.

[21] M. Manceau, J. Gaume, A. Rivaton, J.-L. Gardette, G. Monier, L. Bideux, Thin Solid Films 518 (2010) 7113.

[22] G. Dicker, M. P. de Hass, L. D. A. Siebbeles, Phys. Rev. B 71 (2005) 155204.

[23] H. Ito, Y. Niimi, A. Suzuki, K. Marumoto, S. Kuroda, Thin Solid Films 516 (2008) 2743.

[24] Y. Li, J. Gao, D. Wang, G. Yu, Y. Gao, A. J. Heeger, Synth. Met. 97 (1998) 191.

[25] C. K. Alexander, M. N. O. Sadiku, Fundamentals of Electric Circuits, McGraw-Hill, New York, 2nd edition, 2004.

[26] J. R. Macdonald, Solid State Ion. 13 (1984) 147.

[27] C.-C. Chen, B.-C. Huang, M.-S. Lin, Y.-J. Lu, T.-Y. Cho, C.-H. Chang, K.-C. Tien, S.-H. Liu, T.-H. Ke, C.-C. Wu, Org. Electron. 11 (2010) 1901.

[28] J. Drechsel, B. Mannig, D. Gebeyehu, M. Pfeiffer, K. Leo, H. Hoppe, Org. Electron. 5 (2004) 175.

[29] S. M. Sze, K. K. Ng, Physics of Semiconductor Devices, Wiley, New York, 3rd edition, 2007.

[30] O. Fernandez, D. M. Taylor, J. A. Drysdale, D. M. Ellis, IEEE Trns. Dielectr. Electr. Insul. 13 (2006) 1093.

[31] D. M. Taylor, J. A. Drysdale, I. Torres, O. Fernandez, Appl. Phys. Lett. 89 (2006) 183512.

[32] C. H. Kim, M. H. Choi, S. H. Lee, J. Jang, S. Kirchmeyer, Appl. Phys. Lett. 96 (2010) 123301.

[33] P. Lutsyk, K. Janus, M. Mikolajczyk, J. Sworakowski, B. Boratynski, M. Tlaczala, Org. Electron. 11 (2010) 490. 